\title{
Enhanced Performance for Military Vehicles Using Clustering Algorithm in VANET
}

\author{
Deepak Kumar Malviya \\ M.Tech. Student \\ Department of IT, UIT BU, \\ Bhopal, Madhya Pradesh, India
}

\author{
Prashant Shrivastav \\ Assistant Professor \\ RJIT BSF, Takenpur, Gwalior, \\ Madhya Pradesh India
}

\begin{abstract}
Vehicular Ad-hoc networks are established between mobile vehicles equipped with wireless interfaces that could be either of heterogeneous or homogeneous nature. Vehicles and road side fixed equipment's both of them can be either private (belonging to individuals or companies) or public means of transport service providers. In this network every participating vehicle works as single node or wireless router, allowing cars which are 100 to $300 \mathrm{~m}$ from each other to connect and create a network with a large range. Since vehicle/ node in VANET are quick moving element, so the route among the nodes breaks much of the time. In this paper, clustering performed for the transmission of the data from the source to destination. This technique increases the go with the flow rate of data packets inside the network and
\end{abstract} decreases the end-to-end delay.

KEY WORDS: VANET, Routing Protocols, Heterogenous, Homogeneous nature

\section{INTRODUCTION}

Recent advances in communication technologies have enabled wide range of networks. Vehicular Ad-Hoc Network is one of such network which has received lot of interest in last couple of years. VANET suddenly became an active area of development because of its immense potential to support whole new range of frameworks which will support applications to comfort drivers and passengers. Vehicular Ad-hoc networks are established between mobile vehicles equipped with wireless interfaces that could be either of heterogeneous or homogeneous nature [1]. VANETs (Vehicular Ad-hoc Networks) enable communication of one vehicle with another one as well as in between vehicles and nearby fixed roadside equipment. Vehicles and road side fixed equipment's both of them can be either private (belonging to individuals or companies) or public means of transport (like public service vehicles) service providers. In this network every participating vehicle works as single node or wireless router, allowing cars which are 100 to $300 \mathrm{~m}$ from each other to connect and create a network with a large range. As vehicles go out of the coverage range and get disconnected from the network, other vehicles can join, connecting vehicles to each another creating a mobile internet.

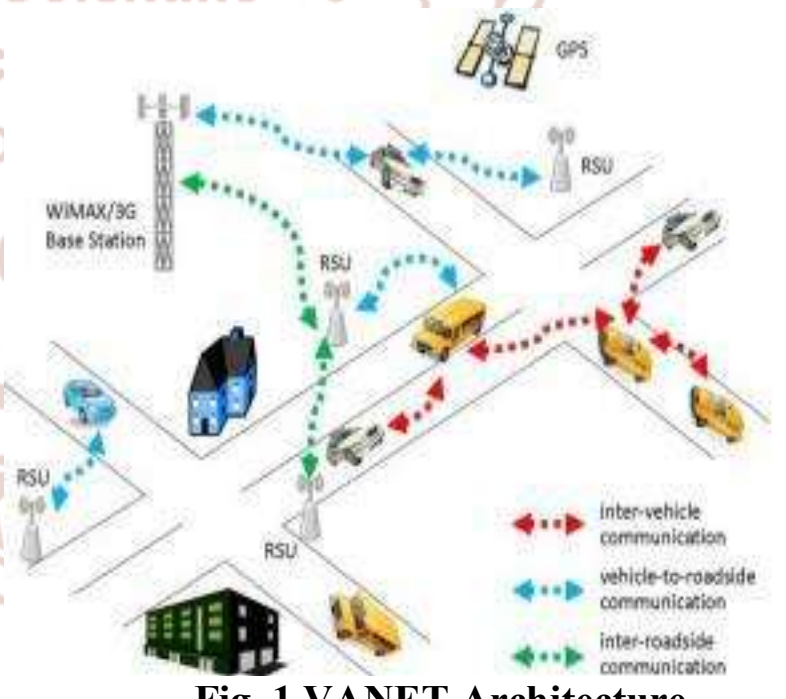

Fig. 1 VANET Architecture

\section{ROUTING PROTOCOL IN VANET}

In VANET, the routing protocols are classified into five categories: Topology based routing protocol, Position based routing protocol, Cluster based routing protocol, Geo cast routing protocol and Broadcast routing protocol. These protocols are characterized on the basis of area / application where they are most suitable [2]. 


\section{A. Topology Based Routing Protocols}

These routing protocols use links information that exists in the network to perform packet forwarding. They are further divided into Proactive and Reactive.

\section{Proactive routing protocols}

The proactive routing means that the routing information, like next forwarding hop is maintained in the background irrespective of communication requests. The advantage of proactive routing protocol is that there is no route discovery since the destination route is stored in the background, but the disadvantage of this protocol is that it provides low latency for real time application. A table is constructed and maintained within a $\mathrm{n}$ ode. So that, each entry in the table indicates the next hop node towards a certain destination. It also leads to the maintenance of unused data paths, which causes the reduction in the available bandwidth. The various types of proactive routing protocols are: LSR, FSR [3].

\section{Reactive/Ad hoc based routing}

Reactive routing opens the route only when it is necessary for an ode to communicate with each other. It maintains only the routes that are currently in use, as a result it reduces the burden in the network. Reactive routing consists of routediscovery phase in which the query packets are flooded into the network for the path search and this phase completes when route is found. $\mathrm{T}$ he various types of reactive routing protocols are AODV, PGB, DSR and TORA

B. Position Based Routing Protocols Position based routing consists of class of routing algorithm. They share the property of using geographic positioning information in order to select the next forwarding hops. The packet is send without any map knowledge to the one hop neighbor, which is closest to destination. Position based routing is beneficial since no global route from source node to destination node need to be created and maintained. Position based routing is broadly divided in two types: Position based greedy V2V protocols, Delay Tolerant Protocols [4].

1. Position Based Greedy V2V Protocols In greedy strategy and intermediate node in the route forward message to the farthest neighbor in the direction of the next destination. Greedy approach requires that intermediate node should possessed position of itself, position of its neighbor and destination position. The goal of these protocols is to transmit data packets to destination as soon as possible that is why these are also known as min delay routing protocols. Various types of position based greedy V2V protocols are GPCR, CAR and DIR

2. Greedy Perimeter Coordinator Routing (GPCR) GPCR is based upon the fact that city street form a natural planner graph. GPCR does not require external static street map for its operation. GPCR consists of two components: A Restricted Greedy forwarding procedure, A repair strategy for routing algorithm. A GPCR follows a destination based greedy forwarding strategy, it routes messages to nodes at intersection. Since GPCR does not use any external static street map so nodes at intersection are difficult to find. GPCR uses heuristic method for finding nodes located at intersections and designates those nodes as coordinators. Coordinator has the responsibility of making routing decisions. There are two approaches used for coordinator determination they are

\section{a) Neighbor Table Approach:}

The nodes periodically transmit beacon messages which contains their position information and last known position information of all neighbors, by listening to beacon messages a $\mathrm{n}$ ode as information about its own position, position of its neighbor and neighbor's neighbor. Using this information node $\mathrm{X}$ consider itself to be within the intersection.

\section{b) Correlation coefficient approach:}

In this case node uses its position information and the position information of its immediate neighbor to find the correlation coefficient, pxy. This approach performs better than neighbor table approach. B y using this approach the algorithm can avoid dependencies on external street map [5].

\section{Connectivity Aware Routing Protocols (CAR):}

CAR protocols find a route to a destination; it has unique characteristics that it maintains the cache of successful route between various source and destination pairs. I $t$ also predicts the position of destination vehicle repairs route as the position changes. Nodes using CAR protocols send periodic Hello beacons that contain their velocity vector information. $\mathrm{O} n$ receiving Hello beacons an ode will record sender in its neighbor table and calculate its own velocity vector and velocity vector of its neighbor. Beacons can also be piggybacked on forwarded data packets to reduce wastage of 
bandwidth and congestion. Entries expire from the neighbor table when the distance between nodes exceeds the threshold value. The CAR protocols establishes the notation of a guard which is a geographic marker message, it is buffered and passed from one vehicle to another to propagate the information. A guard is a temporary message that has an ID, a TTL (Time to live) counts, a radius and some state information. CAR provides two forms of guards. The Standing guard and The Traveling guard. Routing errors may occur due to communication gap between anchor points or due to guards. So CAR protocol has two recovery strategies to cope with the problem. The first strategy is Time out algorithm with active waiting cycle. $\mathrm{T}$ he second strategy is walk around error recovery. $\mathrm{T}$ he CAR protocol has the ability to generate virtual information in the form of guards, which is a distinct advantage over other protocols.

\section{Diagonal-Intersection-Based Routing Protocol (DIR)}

DIR protocol constructs a series of diagonal intersections between the source and destination vehicle. The DIR protocol is based upon the geographic routing protocol in which source vehicle geographically forwards the data packets towards the first diagonal intersection, second diagonal intersection and so on until the last diagonal intersection and finally geographically reaches to designation vehicle. DIR vehicle is auto adjustable, Auto adjustability means that one sub path with low data packet delay between two neighboring diagonal intersections, which is dynamically selected to forward data packets. To reduce the data packet delay the route is automatically selected with lowest sub path delay. DIR protocol can automatically adjust routing path for keeping the lower packet delay [5].

\section{Delay Tolerant Protocols}

In urban scenario where vehicle are densely packed locating a node to carry a message is not a problem but in rural highway situation or in cities at night fewer vehicles are running and establishing end to end route is difficult. So in such cases certain consideration needs to be given in sparse networks. The various types of Delay Tolerant Protocols are MOVE, VADD, and SADV.

\section{Motion Vector Routing Algorithm (MOVE)}

The MOVE algorithm is an algorithm for sparse VANET scenario. I $\mathrm{n}$ these scenarios vehicle act as mobile router that have intermittent connectivity with other vehicles. Connection opportunities must be scrutinized carefully since they occur infrequently and global topology is also rapidly changes. $T$ he algorithm must predict whether forwarding message will provide progress toward intended destination. MOVE algorithm assumes that each node has knowledge of its own position, heading and destination. From this information the current vehicle node can calculate the closest distance between the vehicle and message destination. MOVE algorithm use less buffer space. MOVE algorithm is specially designed for sparse networks and for vehicles that transfer data from sensor networks to base station [5].

\section{Vehicle Assisted Data Delivery (VADD)}

VADD uses a carry and forward strategy to allow packets to be carried by vehicle in sparse networks for forwarding when the node enters the broadcast range, thereby allowing a packet to be forwarded by relay in case of sparse networks. VADD require each vehicle to know its own position and also require an external static street map. Each packet has three modes: Intersection, Straight Way and Destination, where each mode is based on the location of the node carrying the packet. Intersection mode is used when the packet has reached an intersection at which routing decisions can be made for the packet to be forwarded to a vehicle along any of the available directions of the intersection. In Straight Way mode the current node is on a road where there are only two possible directions for the packet to travel, in the direction of the current node or in the opposite direction. Destination mode is when the packet is close to its final destination [6].

\section{Static Node Assisted Adaptive Routing Protocol (SADV)}

SADV aims at reducing message delivery delay in sparse networks. S ADV also dynamically adapts to varying traffic density by allowing each node to measure the amount of time for message delivery. SADV assumes that each vehicle knows its position through GPS and each vehicle has accessed to external static street map. S ADV has three different modules; Static Node Assisted Routing (SNAR), Link Delay Update (LDU) and Multipath Data Dissemination (MPDD). SADV operates in two modes: "In Road Mode" and "Intersection Mode". SNAR make use of optimal paths, which are determined on the basis of graph abstracted from road map. LDU maintains the delay matrix dynamically by 
measuring the delay of message delivery between static nodes. MPDD helps in multipath routing [6].

\section{c) Cluster Based Routing}

Cluster based routing is preferred in clusters. A group of nodes identifies themselves to be a part of cluster and an ode is designated as cluster head will broadcast the packet to cluster. Good scalability can be provided for large networks but network delays and overhead are incurred when forming clusters in highly mobile VANET. I n cluster based routing virtual network infrastructure must be created through the clustering of nodes in order to provide scalability. $\mathrm{T}$ he various Clusters based routing protocols are COIN and LORA_CBF

\section{d) Broadcast Routing}

Broadcast routing is frequently used in VANET for sharing, traffic, weather and emergency, road conditions among vehicles and delivering advertisements and announcements. The various Broadcast routing protocols are BROADCOMM, UMB, VTRADE, and DV-CAST [6].

\section{e) Geo Cast Routing}

Geo cast routing is basically a location based multicast routing. Its objective is to deliver the packet from source node to all other nodes within a specified geographical region (Zone of Relevance ZOR). In Geo cast routing vehicles outside the ZOR are not alerted to avoid unnecessary hasty reaction. Geo cast is considered as a multicast service within a specific geographic region. It normally defines a forwarding zone where it directs the flooding of packets in order to reduce message overhead and network congestion caused by simply flooding packets everywhere. In the destination zone, unicast routing can be used to forward the packet. One pitfall of Geo cast is network partitioning and also unfavorable neighbors, which may hinder the proper forwarding of messages. The various Geo cast routing protocols are IVG, DGCASTOR and DRG [6].

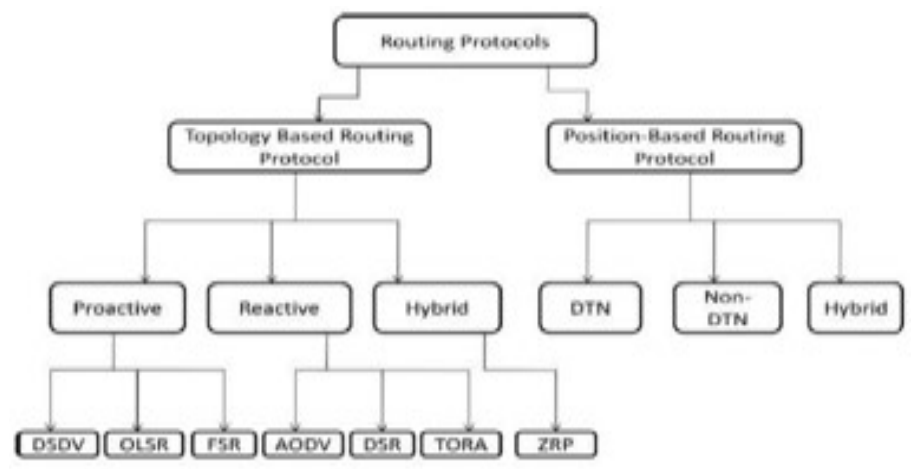

Fig.2 Routing protocol in VANET

\section{LITERATURE SURVEY}

K.LOGESHWARI et al. [2017] in this work, in light of investigation of greedy routing for packet forwarding, we have proposed a Fuzzy Logic based absolutely Greedy Routing (FLGR) protocol. FLGR is a multi- hop routing protocol that is utilized to choose the next-hop node in multi- hop VANETs the utilization of fuzzy logic idea. We have taken into consideration characteristics of a car as an input metrics to fuzzy decision making structures. Based at the best characteristic of simulation consequences, the FLGR successfully choose the fine next-hop node for in addition packet transmission inside the network [7].

Xiaomin Ma et al. [2017] In the plan, a capable new technique coordinating transmitter-situated redundancies, ESD bitmap, message $\mathrm{s}$ sequence number, and modulo based sequence number, smaller than expected opening is given to beat attainable concurrent timer due among close nodes and conceivable message collisions inside the traditional separation based clock for decision of multi-hop forwarders. The unwavering quality and execution of the proposed conspire are analyzed and in correlation with that of various communicate plans for VANET event driven wellbeing administrations on highways. The new analytic account for the effect of the Nakagami blurring channel with separate built up direction loss and vehicle versatility on the reliability and general execution. Extensive simulations are performed to affirm the correctness of our proposed scheme and models below realistic network parameter settings [8].

Shubham Mittal et al.[2016] in this paper, our proposed a technique to development the flexibility of the protocol with the evolving topology. Since vehicle/ node in VANET are quick moving element, so the route among the nodes breaks much of the time. The algorithm builds up a trade course for the data packet transmission when connect break happens. This technique increases the go with the flow rate of data packets inside the network and decreases the endto-end delay. The simulation is accomplished to assess the network performance, and the end result of AODV-AP shows the better PDR and lower network overhead as evaluate to AODV and OLSR [9].

Waqar farooq [2016] et al presented that a novel AUMVs protocol is proposed to develop a VANET among unmanned Military Vehicles (MVs). The proposed protocol performs cluster based multicast 
communication among AUMVs by considering real time and dynamic war field scenario. The AUMVs protocol develops stable clusters and becomes adaptable as indicated by the military environment by using a proposed Priority Based CH Election Scheme (PCHE) amid cluster development which reduces the network overhead and postponement. Additionally, the AUMVs protocol achieves high throughput via combining the multicast technique with a cluster established scheme. The simulation outcome illustrate that the proposed protocol has achieved the purpose of stable and efficient verbal exchange among unmanned MVs.

Pierpaolo Salvo [2016] et al offered that we introduce and inspect a hybrid networking design and protocol which can be used for the efficient execution of this kind of assortment system. We employ a VANETfounded multihop dissemination good judgment to spread manage messages and choose designated nodes. These nodes are exploited to document vehicular data through LTE communications. The performance conduct of the proposed protocol is evaluated via the honour of two actual urban scenarios. In comparing with efficiency bounds that symbolize the efficiency habits attained via state-of the art hybrid integrated VANET and LTE mechanisms, we show our method to offer a vast discount within the traffic load premiums triggered over the LTE cell radio entry process.

\section{PROPOSED MODEL}

In this paper, Weighted Based Clustering Protocol used for the formation of the clusters. Nodes transmit the data or detected events from one cluster head to another cluster head.

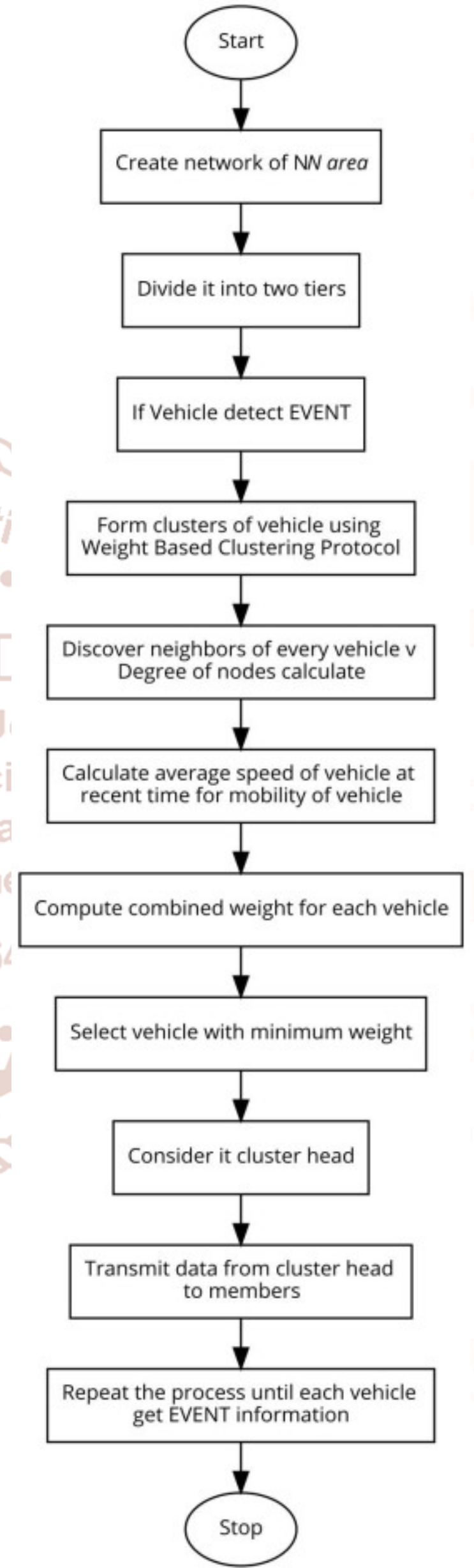

Fig.3 Proposed Flowchart 
International Journal of Trend in Scientific Research and Development (IJTSRD) ISSN: 2456-6470

\section{RESULT ANALYSIS}

For the implementation of the proposed work, we used NS2 for the simulation and the above mentioned techniques are applied to show the work.

Table 1: Parameter Table with their Values

\begin{tabular}{|c|c|}
\hline Parameters & Values \\
\hline Simulation Used & NS2 \\
\hline Network Size & $2000 \mathrm{~m} \mathrm{x} \mathrm{2000} \mathrm{m}$ \\
\hline Number of Nodes & 30 \\
\hline Simulation Time & $20 \mathrm{~s}$ \\
\hline Antenna Used & Omni directional Antenna \\
\hline MAC Protocol & IEEE 802.11 \\
\hline
\end{tabular}

There are three tables which show throughput values, PDR values and Packet drop values at different time period.

Table 2: Throughput

\begin{tabular}{|c|c|c|}
\hline Time (in ms) & Base paper & Propose paper \\
\hline 5 & 5471.9 & 37900.8 \\
\hline 10 & 6092.63 & 37992.7 \\
\hline 12 & 6199.25 & 38073.1 \\
\hline 15 & 6339.68 & 43091.2 \\
\hline 18 & 6429.48 & 46795.5 \\
\hline 20 & 6473.38 & 49581.3 \\
\hline
\end{tabular}

Table 3: Packet Delivery Ratio

\begin{tabular}{|c|c|c|}
\hline Time (in ms) & Base paper & Propose paper \\
\hline 5 & 13.0467 & 26.3893 \\
\hline 10 & 12.9467 & 25.9971 \\
\hline 12 & 12.9166 & 25.8421 \\
\hline 15 & 12.9089 & 26.2179 \\
\hline 18 & 12.8912 & 26.073 \\
\hline 20 & 12.895 & 25.9037 \\
\hline
\end{tabular}

Table 4: Routing Overhead

\begin{tabular}{|c|c|c|}
\hline Time (in ms) & Base paper & Propose paper \\
\hline 5 & 25817 & 19057 \\
\hline 10 & 53335 & 34045 \\
\hline 12 & 64398 & 43488 \\
\hline 15 & 81224 & 56634 \\
\hline 18 & 98042 & 72807 \\
\hline 20 & 108553 & 84206 \\
\hline
\end{tabular}

\section{CONCLUSION}

VANETs enable communication of one vehicle with another one as well as in between vehicles and nearby fixed roadside equipment. Vehicles and road side fixed equipment's both of them can be either private or public means of transport service providers. In
VANET surroundings every vehicle acts a mobile node and for exchanging data it acts a source or a destination or a router. Although VANET is a sub category of MANET however its nature is dynamic due to pace of mobile nodes. With the proposed work, the clustering performed to enhance the network lifetime and decrease the delay of the data.

\section{REFERENCES}

1. Mrunmayi S Sahasrabudhe, Dr. Meenu Chawla "Survey of Applications based on Vehicular AdHoc Network (VANET) Framework" / (IJCSIT) International Journal of Computer Science and Information Technologies, Vol. 5 (3) , 2014, 3937-3942.

2. James Bernsen, D. Mnivannan, "Unicast routing protocols for vehicular ad hoc networks: A critical comparison and classification", in journal of Pervasive and Mobile Computing 5 (2009) 1-18

3. Jetzabel Serna, Jesus Luna and Manel Medina "Geolocation-based Trust for Vanet's Privacy" Dynamic Publishers, Journal of Information Assurance and Security 4 (2009) Page 432-439, 2009

4. J. Angel F. Lage, C. Pereiro Gestoso, O. Rubinos, F. Aguado Agelet "Analysis of Unicast Routing Protocols For VANETs" 2009 Fifth International Conference on Networking and Services

5. Kargal, F. Papadimitratos, P. Buttyan, L. Muter, M. Schoch, E. Wiedersheim, B. Ta-Vinh Thong Calandriello, G. Held, A. Kung, A. Hubaux, J. -P. Ulm Univ., Ulm "Secure Vehicular Communication Systems: Implementation, Performance and Research Challenges", IEEE Communication Magazine, Vol. 46 issue: 11, November 2008

6. T. Sawamura, K. Tanaka, M. Atajanov, N. Matsumoto, and N. Yoshida, “Adaptive Router Promotion and Group Forming in Ad hoc Networks", International Journal of Ad hoc and Ubiquitous Computing (IJAHUC), Vol 3, no 4, 2008, pp 217-223

7. K. LOGESHWARI, Dr. L. LAKSHMANAN, M.E "Authenticated anonymous secure on demand routing protocol in VANET (vehicular ad hoc network)" international conference on information, communication \& embedded systems (ICICES 2017). 
International Journal of Trend in Scientific Research and Development (IJTSRD) ISSN: 2456-6470

8. Xiaomin Ma, Gabe Kanelopoulos, and Kishor S. 10. waqar farooq, Muazzam Ali Khan and Saad Trivedi "Application-level Scheme to Enhance VANET Event-driven Multi-hop Safety-Related Services" 2017 Workshop on Computing, Networking and Communications (CNC)

9. Shubham Mittal, Ramandeep Kaur and Kamlesh C. Purohit "Enhancing the Data Transfer rate by Creating Alternative Path for AODV Routing Protocol in VANET" 978-1-5090-34802/16/\$31.00 C2016 IEEE. Rehman, "A Cluster based Multicast routing protocol for Autonomous Unmanned Military Vehicles (AUMVs) communication in VANET", 978-1-5090-1252-7/16/\$31.00 C2016 IEEE.

11. Pierpaolo Salvo, Ion Turcanu, Francesca Cuomo, Andrea Baiocchi and Izhak Rubiny, "LTE Floating Car Data application off-loading via VANET driven clustering formation", ISBN 9783-901882-79-1 C 2016 IFIP.

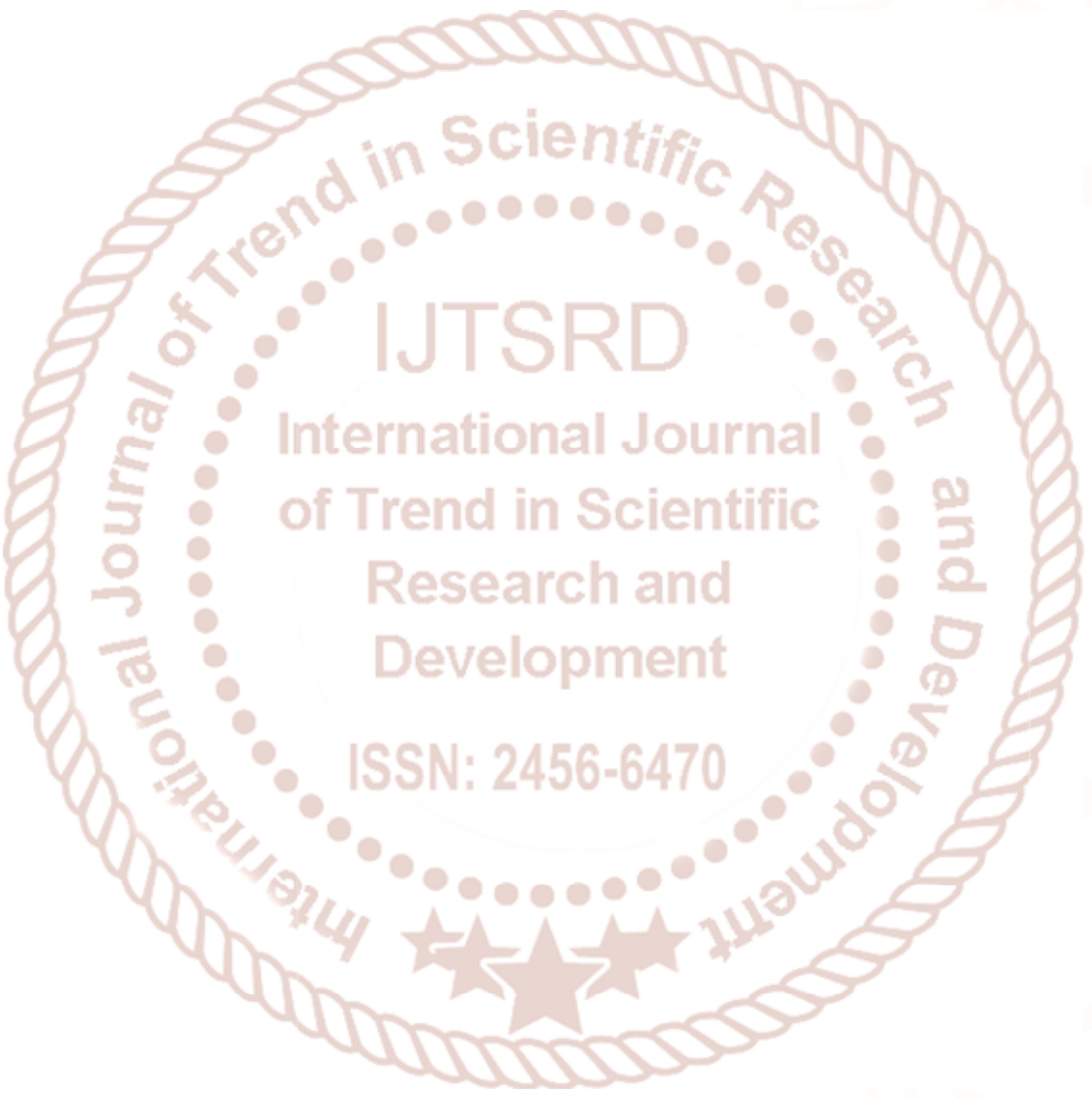

